\title{
POI-0I. The blood transcriptional response to early acute HIV infection is transient and responsive to antiretroviral therapy
} JA Skinner*1, M Sharma1 ${ }^{1}$ N Baldwin ${ }^{1}$, B Lemoine ${ }^{1}$, D Blankenship ${ }^{2}$, EB De $\mathrm{Vol}^{2}$, A Mejias 3 , O Ramilo3 ${ }^{3}$ M Cohen ${ }^{4}$, N Letvin' ${ }^{5}$, D Goldstein ${ }^{6}$, K Soderberg7, TN Denny ${ }^{6}$, K Shianna ${ }^{6}$, A McMichael ${ }^{8}$, B Haynes ${ }^{7}$, $\mathrm{J} \mathrm{Banchereau}^{1}$ and D Chaussabel ${ }^{1}$

Address: ${ }^{1}$ Baylor Institute for Immunology Research, Dallas, TX, USA, ${ }^{2}$ Baylor Health Care System, Dallas, TX, USA, ${ }^{3}$ Dept. of Pediatrics, UTSW, Dallas, TX, USA, ${ }^{4}$ University of North Carolina, Chapel Hill, NC, USA, ${ }^{5}$ Beth Israel Deaconess Medical Center, Harvard Medical School, Boston, MA, USA, ${ }^{6}$ Duke Institute for Genome Sciences and Policy, Duke University, Durham, NC, USA, ${ }^{7}$ Duke University School of Medicine, Durham, $\mathrm{NC}$, USA and ${ }^{8}$ Weatherall Institute of Molecular Medicine, Oxford, UK

* Corresponding author

from AIDS Vaccine 2009

Paris, France. 19-22 October 2009

Published: 22 October 2009

Retrovirology 2009, 6(Suppl 3):PI doi:I0.I 186/I742-4690-6-S3-PI

This abstract is available from: http://www.retrovirology.com/content/6/S3/PI

(c) 2009 Skinner et al; licensee BioMed Central Ltd.

\section{Background}

Systemic events in acute HIV infection (AHI) are associated with disease severity and progression to AIDS. Timely identification of AHI patients has posed a significant challenge to identifying the underlying mechanisms driving these events. The aim of this study is to elucidate these pathways by characterizing the genome-wide transcriptional signature expressed by whole blood during early AHI.

\section{Methods}

Longitudinal whole blood samples from ART treated and untreated patients from both the United States $(n=16)$ and Africa $(\mathrm{n}=16)$ were collected at study enrollment and weeks $1,2,4,12$, and 24 . AHI and non-infected controls were analyzed using Illumina HT-12 microarrays. Both gene and module level analysis were conducted to identify biologic pathways active in AHI.

\section{Results}

Nineteen annotated and 24 undefined transcriptional modules constitute a robust transcriptional signature that collectively distinguished early AHI patients from noninfected controls. The activity of transcriptional modules related to interferon, cell cycle, cytotoxic, and mitochon- drial responses were significantly increased in AHI patients. At study enrollment, the intensity of this signature was not correlated with viral load and exhibited heterogeneity between patients. Association between viral load and signature intensity was found over time. However, three patients exhibited little change in transcriptional activity despite high viral loads. When compared to acute RSV and Influenza infections, only interferon signatures were conserved across all three infections while cell cycle, cytotoxic, and mitochondrial responses were unique to AHI. The AHI signature of untreated patients regressed to non-infected control levels by 12-24 weeks post enrollment. The initiation of ART accelerated the dissipation of this signature, returning the core AHI signature to normal levels within 4 weeks.

\section{Conclusion}

The whole blood AHI transcriptional signature is unique, transient, and capable of classifying individual responses to infection. This signature is responsive to ART and contains pathways with both defined and novel associations with HIV infection. 